\title{
The anchoring of gaseous jet diffusion flames in stagnant air
}

\author{
Vadim Kurdyumov ${ }^{\mathrm{a}}$, Eduardo Fernández-Tarrazo ${ }^{\mathrm{b}, *}$, \\ Amable Liñán ${ }^{\mathrm{c}}$ \\ ${ }^{a}$ Instituto Tecnológico de Veracruz, Veracruz, Mexico \\ b Instituto Nacional de Técnica Aeroespacial, Madrid, Spain \\ ${ }^{c}$ E.T.S.I. Aeronáuticos, UPM, Madrid, Spain
}

\begin{abstract}
A numerical analysis is presented to describe the structure of the attachment region of gaseous jet diffusion flames in stagnant air. For the typical Reynolds numbers encountered in applications, the region of flame attachment, in the near wake at the injector rim, is small compared with the injector radius. The local flow, which we consider to be laminar, quasi-steady and two-dimensional, is determined by the wall value of the fuel stream velocity gradient and the chemical reaction time or residence time in the premixed flame of a stoichiometric mixture of the fuel stream and required amount of air. When the Karlovitz number, or product of the velocity gradient and the flame residence time, grows above a critical value the flame is lifted off the injector.
\end{abstract}

Keywords: Flame attachment; Diffusion flame; Flame anchoring; Jet combustion; Methane flame

\section{Introduction}

In many combustion systems the fuel and the oxidizer enter the combustion chamber in separate streams, so that mixing and chemical reaction occur simultaneously.

If the relevant chemical reaction time is small compared with the mixing time the fuel and the oxygen will only coexist, with small concentrations, in thin reaction layers, or diffusion flames; there they react with a rate determined by the rate at which the reactants diffuse to the flame. Because the overall combustion reactions are exothermic the temperature distribution in the thin reaction layer shows peak values which are close to the adiabatic flame temperature $T_{e}$ of a stoichiometric mixture formed with the fuel and oxidizer feed streams. The relevant reaction time, which must be small compared with the diffusion time to avoid local quenching of the diffusion flame, corresponds to the adiabatic flame temperature and can be identified as the residence time in the premixed flame of the stoichiometric mixture. The relevant diffusion time is the inverse of the scalar dissipation at the reaction layer.

\footnotetext{
${ }^{*}$ Corresponding author.

E-mail address: fdezte@inta.es (E. Fernández-Tarrazo).
}

However, due to the large sensitivity with temperature of the effective rates of the overall combustion reactions, the rate of the reaction at the lower temperature of the reactants in their feed streams, with non-hypergolic fuels, will be too small for the reaction to occur in the combustion chamber. In order to trigger the reaction we must use an external local heat source to generate a premixed flame front in the partially mixed flow field. The premixed flame will have lean and rich branches where the fuel or the oxygen will be completely consumed, and will leave behind a trailing diffusion flame originating in the stoichiometric surface. The triple flame front may be blown off the chamber if the flow velocity is higher than the front velocity; otherwise, it may move up to a stationary lifted flame position or, if possible, all the way up to the fuel injector rim, leaving behind a diffusion flame attached to the fuel injector.

The purpose of this paper is to contribute to the analysis of the structure of the attachment region of diffusion flames, and to the conditions for lift-off, by means of a detailed description of the attachment region of fuel jet diffusion flames in stagnant air.

The problem of determining the conditions of attachment of diffusion flames to the injector rim has received considerable attention in the literature. See, for example, the early 


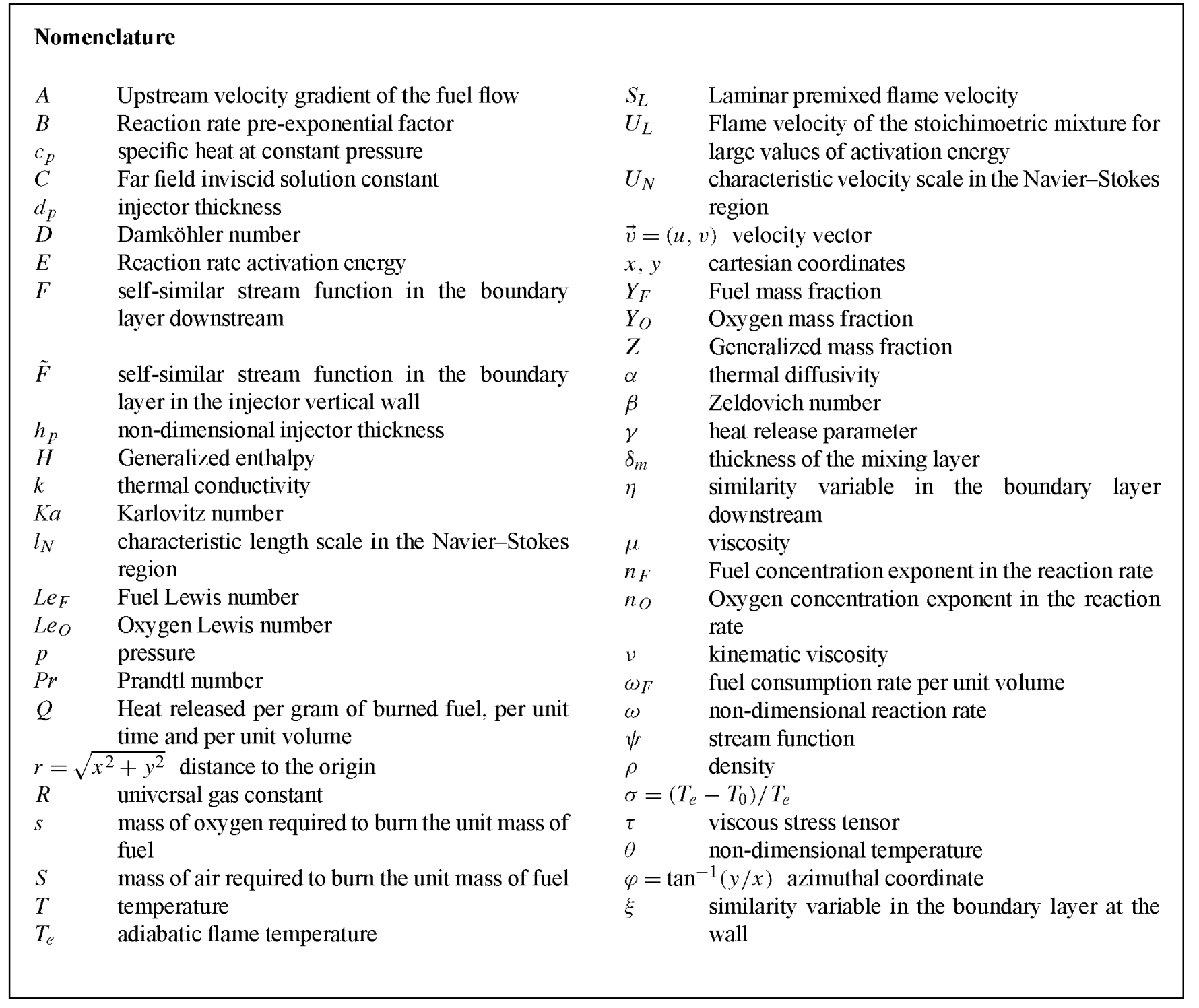

work reviewed in the book by Gaydon and Wolfhard [3] and the more recent work of Robson and Wilson [10], Kawamura and Asato [6], Kawamura et al. [7] and, particularly, the extensive work carried out and reviewed by Takahashi and Schmoll [13] and Takahashi et al. [14].

The size of the flame attachment region is very small in high Reynolds number flows of typical jet diffusion flames, and this contributes to the difficulties of the experimental description. When trying to describe this region using numerical techniques we should take into account that mixing between the fuel stream and the stagnant, or coflowing, air takes place in a thin anular mixing layer between the streams; which develops downstream of the injector rim when the fuel and air boundary layers on the wall of the injector meet.

In the mixing layer downstream of the small flame attachment region at the rim of the injector upstream diffusion and conduction can be neglected, although they must be retained when describing the premixed flame structure and propagation of the triple flames along the mixing layer during the ignition transient.

The essential role of upstream heat conduction and diffusion in the attachment of diffusion flames was recognized by Gaydon and Wolfhard [3], who indicated that close to the injector there is a region where the reactants are partially mixed. This region is bounded downstream by a premixed flame, prevented to propagate upstream by the flow and the heat losses to the injector. The formulation of the problem of determining the conditions for diffusion flame attachment was advanced by Liñán [8] and, in more detail, by Fernández-Tarrazo et al. [2] where preliminary results of the numerical description are given.

The first analysis of the laminar mixing layer between two parallel streams, initially separated by a splitter plate here the injector wall - was carried out by Goldstein [4], using the boundary layer approximation. When the fuel and air streams first meet downstream of the splitter plate they develop a mixing layer which close to the injector is thin com- 
pared with the fuel and air boundary layers at the injector wall. In the Goldstein description the mixing layer structure is self-similar, determined by the kinematic viscosity $v$ and the wall velocity gradient $A$ (for example, of the fuel boundary layer). In the Goldstein description the thickness $\delta_{m}$ of the mixing layer grows with the downstream distance $x$ from the injector as $(x \nu / A)^{1 / 3}$ and the velocity as $A(x \nu / A)^{1 / 3}$. The boundary layer approximation is applicable only as long as $\delta_{m} / x$ is small compared with unity, at distances $x$ from the injector large compared with the size $\left(\nu_{0} / A\right)^{1 / 2}$ of the Navier-Stokes region. This is the position where the two velocity components are of the same order, $\left(v_{0} A\right)^{1 / 2}$, such that the local Reynolds number is of the order unity, and the region where the attached diffusion flame originates.

In the mixing layer the transverse velocity component, of the order $\left(v_{0}^{2} A / x\right)^{1 / 3}$, is an entrainment velocity associated with the growth with $x$ of the $u$ component of velocity. It is a positive entrainment velocity if we ignore the dilatation effects resulting from the heat release, associated with a diffusion controlled reaction in the mixing layer; these dilatation effects may lead to a positive displacement velocity of the gas mixture outside the mixing layer. Stewartson [12] and Messiter [9] showed the existence of a triple deck structure in a region close to the trailing edge of the splitter plate where we find spatial pressure variations associated with the entrainment (or displacement) velocities. When there is a diffusion flame in the mixing layer, we may find overpressures that change the wall values of the velocity gradients in the fuel and air boundary layers and, thus, also the boundary conditions of the flow in the Navier-Stokes region of attachment of the diffusion flame, as shown in Higuera and Liñán [5].

However, in the particular case which we consider in this paper where the fuel jet discharges into stagnant air, the overpressures needed to bring the air to the mixing layer are too small to affect the fuel flow outside the mixing layer, and the triple deck structure is absent.

When observing the flow at the scale of the NavierStokes region of attachment of the flame, the external flow on the fuel side is seen as a uniform shear flow with the wall velocity gradient of the fuel stream -even if the fuel stream is a fully developed parabolic flow-. The velocity gradient $A$ and the thermal diffusivity $\alpha_{0}=v_{0} / \operatorname{Pr}$ of the fuel stream will determine the characteristic scales $l_{N}=\sqrt{\alpha_{0} / A}$ and $U_{N}=\sqrt{\alpha_{0} A}$ for the length and the velocity in the region. The thickness $d_{p}$ of the injector wall, when measured with $l_{N}$, leads to a parameter $h_{p}=d_{p} / l_{N}$ which will certainly play a role in the attachment of the diffusion flame. Here we shall consider only the two limiting cases: (a) $h_{p} \rightarrow \infty$, corresponding to a fuel jet coming out flush from a wall into stagnant air, and (b) $h_{p} \rightarrow 0$, corresponding to an injector with an infinitely thin wall surrounded by stagnant air.

Although in this work we will consider the flow near to the injector to be laminar and quasi-steady, the results may be qualitatively correct as well when dealing with the more realistic turbulent flows. This may expected to be so because the size of the flame attachment region in the turbulent case will again be determined by the wall value of the shear and the condition that the local Reynolds number is of order unity, to allow for upstream conduction and diffusion. But in the description of the mean values of the turbulent boundary layer these conditions determine the friction velocity and the thickness of the viscous sublayer; this sublayer is transformed directly without change in scale into the flame attachment region at the rim of the injector. In this region the Reynolds stresses are no longer dominant; we can anticipate that the main effect of turbulence is to introduce time variations in the wall velocity gradient perhaps without significant changes in the physics of the problem

\section{Formulation}

As indicated above, we shall analyze the laminar flow in the near wake region of the fuel injector which is locally two-dimensional and quasi-steady, forced by a uniform shear flow with velocity gradient $A$ on the fuel side and stagnant air on the other side.

The chemical reaction between the fuel and oxygen of the air will be modelled by an overall irreversible reaction, such that $s$ grams of oxygen are consumed and $(1+s)$ grams of products are generated together with a thermal energy release $Q$ per gram of fuel consumed. For the fuel consumption rate per unit volume $\omega_{F}$ we shall use an Arrhenius reaction rate of the form

$\omega_{F}=B \rho^{n_{F}+n_{O}} Y_{F}^{n_{F}} Y_{O}^{n_{O}} \exp (-E / R T)$,

involving the pre-exponential factor $B$, the density $\rho$, the fuel and oxygen mass fractions $Y_{F}$ and $Y_{O}$, respectively, the activation temperature $E / R$, the temperature $T$, and the reaction orders $n_{F}$ and $n_{O}$ of the fuel and oxygen, respectively. The calculations in this paper will be carried out with the values suggested by Westbrook and Dryer [15] $-n_{F}=0.2$ and $n_{O}=1.3-$ for the combustion of methane and air. Notice that in this case, because $n_{F}<1$, we must complement (1) with the condition $\omega_{F}=0$ if $Y_{F} \leqslant 0$ to ensure that non-negative values of $Y_{F}$ will be obtained in the calculations.

In the calculations we will consider the Prandtl number to be constant $P r=0.72$, and the Lewis numbers $L e_{F}=L e_{O}=$ 1 for simplicity. In the formulation we shall also consider the specific heat $c_{p}$ and the mean molecular mass of the mixture to be constant. We shall use a power law dependence with $T$ of the coefficient of viscosity $\mu / \mu_{0}=\left(T / T_{0}\right)^{1 / 2}$. The equations describing the low Mach number steady flow in the Navier-Stokes region will be written in non-dimensional form using $l_{N}$ and $U_{N}$ as scales for the spatial coordinates and for the velocity $\vec{v} ; \rho_{0} U_{N}^{2}$ is used as scale for the pressure variations $p, \rho_{0}$ for the density and $\mu_{0}$ for the viscosity. The fuel and oxygen mass fractions $Y_{F}$ and $Y_{O}$ will be 
normalized with their values $Y_{F 0}$ and $Y_{O 0}$ in the fuel stream and in the stagnant air. Then, the equations assume the form

$$
\begin{aligned}
& \nabla \cdot(\rho \vec{v})=0, \\
& \nabla \cdot(\rho \vec{v} \vec{v})=-\nabla p+\operatorname{Pr} \nabla \cdot \tau^{\prime}, \\
& \nabla \cdot\left(\rho \vec{v} Y_{F}\right)=\frac{1}{L_{F}} \nabla \cdot\left(\mu \nabla Y_{F}\right)-\omega, \\
& \nabla \cdot\left(\rho \vec{v} Y_{O}\right)=\frac{1}{L_{O}} \nabla \cdot\left(\mu \nabla Y_{O}\right)-S \omega, \\
& \nabla \cdot(\rho \vec{v} \theta)=\nabla \cdot(\mu \nabla \theta)-(1+S) \omega, \\
& \mu^{2}=1 / \rho=(1+\gamma \theta) .
\end{aligned}
$$

Here $\tau_{i j}^{\prime}=\mu\left(\nabla_{i} v_{j}+\nabla_{j} v_{i}\right)$ is the viscous stress tensor because the term $\left(\mu_{v}-\frac{4}{3} \mu\right) \nabla_{k} v_{k} \delta_{i j}$ can be absorbed in the pressure variations, and $\theta=\left(T-T_{0}\right) /\left(T_{e}-T_{0}\right)$ is the nondimensional temperature rise based on the initial value $T_{0}$ of the temperature in the fuel stream and in the air, considered for simplicity to be equal. $T_{e}=T_{0}+Q Y_{F 0} / c_{p}(1+S)$ is the adiabatic flame temperature of the stoichiometric fuel and air mixture; $\gamma=Q Y_{F 0} / C_{p} T_{0}(1+S)=\left(T_{e}-T_{0}\right) / T_{0}$ is the heat release parameter and $S=s Y_{F 0} / Y_{O 0}$ is the mass of air required to burn the unit mass of the fuel stream. The Prandtl number appears here in the momentum equation because the scales $l_{N}$ and $U_{N}$ are based on the thermal diffusivity.

The non-dimensional reaction rate is given by

$$
\begin{aligned}
\omega= & D \rho^{n_{F}+n_{O}} Y_{F}^{n_{F}} Y_{O}^{n_{O}} \\
& \times \exp (\beta(\theta-1) /(1+\sigma(\theta-1))),
\end{aligned}
$$

where

$$
D=\rho_{0}^{n_{F}+n_{O}-1} B Y_{O 0}^{n_{O}} Y_{F 0}^{n_{F}-1} \exp \left(-E / R T_{e}\right) / A
$$

is a Damköhler number, $\beta=E\left(T_{e}-T_{0}\right) / R T_{e}^{2}$ is the Zeldovich number, and $\sigma=\left(T_{e}-T_{0}\right) / T_{e}=\gamma /(1+\gamma)$. Although the Damköhler number $D$ in Eq. (3) appears naturally when writing the Arrhenius reaction rate in nondimensional form, we shall present the results in terms of an equivalent Damköhler number defined as the ratio of the residence time $1 / A$ in the Navier-Stokes region and the transient time $\alpha_{0} / S_{L}^{2}$ in the premixed flame associated with the stoichiometric mixture formed by 1 gram of the fuel stream and $S$ grams of air (the upstream nonscaled values of the mass fractions of fuel and oxygen are $Y_{F u}=Y_{F 0} /(1+S)$ and $Y_{O u}=Y_{O 0} S /(1+S)$, respectively, and the flame temperature is $\left.T_{e}=T_{0}+Q Y_{F 0} / c_{p}(1+S)\right) . S_{L}$ is the laminar flame velocity of this stoichiometric mixture. The new Damköhler number $S_{L}^{2} / \alpha_{0} A$ is equal to the inverse of the Karlovitz number,

$K a=\alpha_{0} A / S_{L}^{2}$,

which is the appropriate measure of the fuel flow velocity gradient to characterize the flame attachment process.

For large values of the activation energy, $S_{L}$ can be replaced by

$$
\begin{aligned}
U_{L}= & \left(2 \tilde{\Gamma} \frac{\rho_{e}^{n_{F}+n_{O}}}{\rho_{0}^{2}} \frac{k_{e}}{c_{p}}\right. \\
& \left.\times \frac{B Y_{O u}^{n_{o}} Y_{F u}^{n_{F}}-1 \exp \left(-E / R T_{e}\right)}{\beta_{u}^{n_{F}+n_{O}+1}} L_{O}^{n_{O}} L_{F}^{n_{F}}\right)^{1 / 2} .
\end{aligned}
$$

Here $\tilde{\Gamma}$ is the value of the complete Gamma function of the order $n_{F}+n_{O}+1, \tilde{\Gamma}=\Gamma_{n_{F}+n_{O}+1}$. The resulting value of the effective Damköhler number $\mathrm{Ka}^{-1}$ is given in terms of $D$ by

$$
\begin{aligned}
K a^{-1}= & \left(U_{L} / U_{N}\right)^{2} \\
= & \frac{2 \tilde{\Gamma} D}{(1+\gamma)^{n_{F}+n_{O}-1 / 2} \beta^{n_{F}+n_{O}+1}} \\
& \times \frac{S^{n_{O}}}{(1+S)^{n_{F}+n_{O}-1}} .
\end{aligned}
$$

We expect that the results obtained for Arrhenius reactions will be applicable to the real kinetics when represented in terms of the effective Damköhler number $\mathrm{Ka}^{-1}$; particularly, if we choose the reaction orders, the pre-exponential factor and the activation energy of the overall reaction, as Westbrook and Dryer [15] did, to mimic the experimental variation of the premixed flame velocity with the upstream values of the equivalence ratio.

\section{Boundary conditions and asymptotic form of the solution}

They will be given here for the case a) of an infinitely large value of the non-dimensional ratio $h_{p}=d_{p} / l_{N}$-or, equivalently, for a fuel jet issuing from a wall into stagnant air. We shall use the coordinates sketched in Fig. 1.

The boundary conditions on the injector wall are $u=$ $v=0, \theta=0$, together with $\partial Y_{F} / \partial y=\partial Y_{O} / \partial y=0$ for $y=0, x<0$ and $\partial Y_{F} \partial x=\partial Y_{O} / \partial x=0$ for $x=0, y<0$.

Notice that we consider that there is no significant change in the injector wall temperature associated with the heat received by the injector from the flame. This is well justified

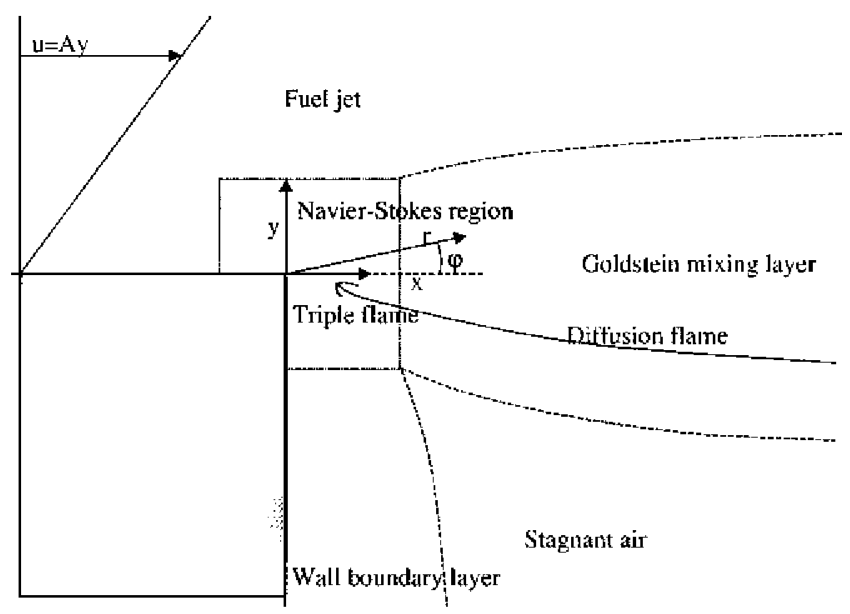

Fig. 1. Sketch of the problem. 
if metallic injectors are used. In this case heat received by conduction from the flame is rapidly conducted upstream through the injector and lost to the boundary layer of the fuel stream.

For large values of the distance $r=\left(x^{2}+y^{2}\right)^{1 / 2}$ to the origin (which is measured with the scale $l_{N}$ ), the values of $Y_{F}-1, Y_{O}, \theta, v$ and $u-y$ on the fuel side $y>0$ must tend to their values equal to 0 in the fuel stream.

On the air side, $y<0, Y_{F}, Y_{O}-1, \theta, v$ and $u$ must also tend to 0 . The decay to zero of the perturbations of the flow variables on the fuel stream is fast, due to the linear growth with $y$ of the $u$ component of the velocity. However, the decay of the flow velocity to zero on the originally stagnant air side is very slow. The slow decay to zero of the perturbations on the air side must be taken into account in the boundary conditions when solving numerically the conservation equations to ensure accuracy in the results. The motion on the air side is due to the air entrainment by viscous forces in the mixing layer, so that we shall begin the analysis by a description of the mixing layer accounting for the thermal expansion effects associated with the diffusion controlled combustion. The analysis will provide us with the value of the entrainment velocity on the air boundary of the mixing layer. This velocity, which varies with $x$ as $x^{-1 / 3}$, determines the flow on the air side which is irrotational and decays with $r$ as $r^{-1 / 3}$ outside a rotational boundary layer adjacent to the injector wall. Thus, we begin our description of the far field, needed for our numerical analysis of the region of flame attachment, with a description of the mixing layer which drives the air entrainment flow.

At large values of $r$, and with $\eta=y / x^{1 / 3}$ of the order unity, the asymptotic value of the solution must correspond to the self-similar solution of the boundary layer form of the conservation equations, in the limit of large local Damköhler numbers. This is so because, even though the appropriate Damköhler number $\mathrm{Ka}^{-1}$ in the Navier-Stokes region may be of the order unity (to ensure the chemical time $\alpha_{0} / S_{L}^{2}$ to be at most of the order of the local residence time $1 / A)$, the Damköhler number in the downstream mixing layer will grow with $x^{2 / 3}$, rapidly becoming large compared with unity. Then, in the Goldstein mixing layer the reaction will be diffusion controlled. The solution of this problem which corresponds to a generalization of the Goldstein pure fluid dynamic mixing layer problem - will be given here in the particular case of unity Lewis number. In this case we can replace the conservation equations for $Y_{F}, Y_{O}$ and $\theta$ by the (Burke-Schumann) chemical equilibrium condition, $Y_{F} Y_{O}=0$, of non-coexistence of the fuel and oxygen, together with two conservation equations for the SchvabZeldovich variables: the mixture fraction $Z=\left(S Y_{F}-Y_{O}+\right.$ 1) $/(1+S)$ and the excess enthalpy $H=Y_{F}+Y_{O}+\theta-1$ which satisfy the conservation equations free from chemical reaction terms, obtained by linear combinations of the original equations.

For values of $x$ large compared with unity, and $\eta=$ $y / x^{1 / 3}$ of the order unity, the asymptotic form of the solution of the conservation equations is, like in the Goldstein compressible flow description, a self-similar solution of the boundary layer form of the equations given by $\psi=$ $x^{2 / 3} F(\eta), Z=Z(\eta)$ and $H=H(\eta)$. Here $\psi$ is the stream function defined by $\rho u=\psi_{y}, \rho v=-\psi_{x}$. The resulting system of equations are

$\frac{1}{3} \rho U^{2}-\frac{2}{3} F U^{\mathrm{I}}=\operatorname{Pr}\left(\mu U^{\mathrm{I}}\right)^{\mathrm{I}}$,

$-\frac{2}{3} F Z^{\mathrm{I}}=\left(\mu Z^{\mathrm{I}}\right)^{\mathrm{I}}$

$-\frac{2}{3} F H^{\mathrm{I}}=\left(\mu H^{\mathrm{I}}\right)^{\mathrm{I}}$,

where $\rho U(\eta)=F^{\mathrm{I}}(\eta)$, and the boundary conditions

$$
\begin{aligned}
U(-\infty) & =U(+\infty)-\eta=Z(-\infty)=Z(+\infty)-1 \\
& =H(-\infty)=H(+\infty)=0 .
\end{aligned}
$$

With these boundary conditions the solution of equation (9) is given by $H \equiv 0$. The numerical solution of the remaining system of equations leads to the results shown in Fig. 2 for $S=17.4$ corresponding to a jet of pure methane. Notice that because of the large values of $S$ the flame lies well on the oxidizer side. The value $F(-\infty)$ determines, through the entrainment velocity $v=-\frac{2}{3} x^{-1 / 3} F(-\infty)$ at the edge of the mixing layer, the weak flow generated on the otherwise stagnant air. The resulting negative value of $F(-\infty)$ is not large and, due to the high diffusivities at the high combustion temperatures, allows the fuel to penetrate deep into the air side of the mixing layer. The large values of $(-\eta)$ where the flame lies are also associated with the large values of $S$, which implies that a large amount of air is required to burn the unit mass of fuel.

In order to calculate the decay with $r$ of the perturbation outside the mixing layer, we can proceed with the theory of asymptotic expansions as was done by FernándezTarrazo et al. [2]. On the air side of the mixing layer we find an entrainment velocity associated with values of the stream function $\psi$ given by $\psi=F(-\infty) x^{2 / 3}$. This entrainment velocity generates in the region $r \gg 1,-\pi / 2<\varphi<0$ an inviscid irrotational flow with $\psi=C r^{2 / 3} \sin \frac{2}{3}\left(\varphi+\frac{\pi}{2}\right)$, where $(C=2 / \sqrt{3} F(-\infty))$, as obtained by matching for $\varphi \rightarrow 0$ with the solution at the downstream boundary layer for $\eta \rightarrow-\infty$. Near to the wall, when $\varphi+\frac{\pi}{2} \rightarrow 0, \psi \rightarrow$ $(-y)^{2 / 3} \frac{2}{3} C \frac{x}{(-y)}$, there is a non-zero tangential velocity of the order $(-y)^{-1 / 3}$; so that we encounter a boundary layer adjacent to the wall, which must be described to show how the tangential velocity decreases to zero when approaching the wall. The thickness of the boundary layer turns out to be of the order $(-y)^{2 / 3}$, and the flow has a self-similar description given by $\psi=(-y)^{1 / 3} \sqrt{\frac{2 C}{3}} \tilde{F}\left(\sqrt{\frac{2 C}{3}} \frac{x}{(-y)^{2 / 3}}\right)$. Here $\tilde{F}(\xi)$ satisfies the equation $\tilde{F}^{\mathrm{I}} \tilde{F}^{\mathrm{II}}+\frac{1}{3} \tilde{F} \tilde{F}^{\mathrm{III}}-\operatorname{Pr} \tilde{F}^{\mathrm{IV}}=0$, with boundary conditions given by $\tilde{F}(0)=\tilde{F}^{\mathrm{I}}(0)=0$ at the wall, and the asymptotic behaviour of the solution, for large 


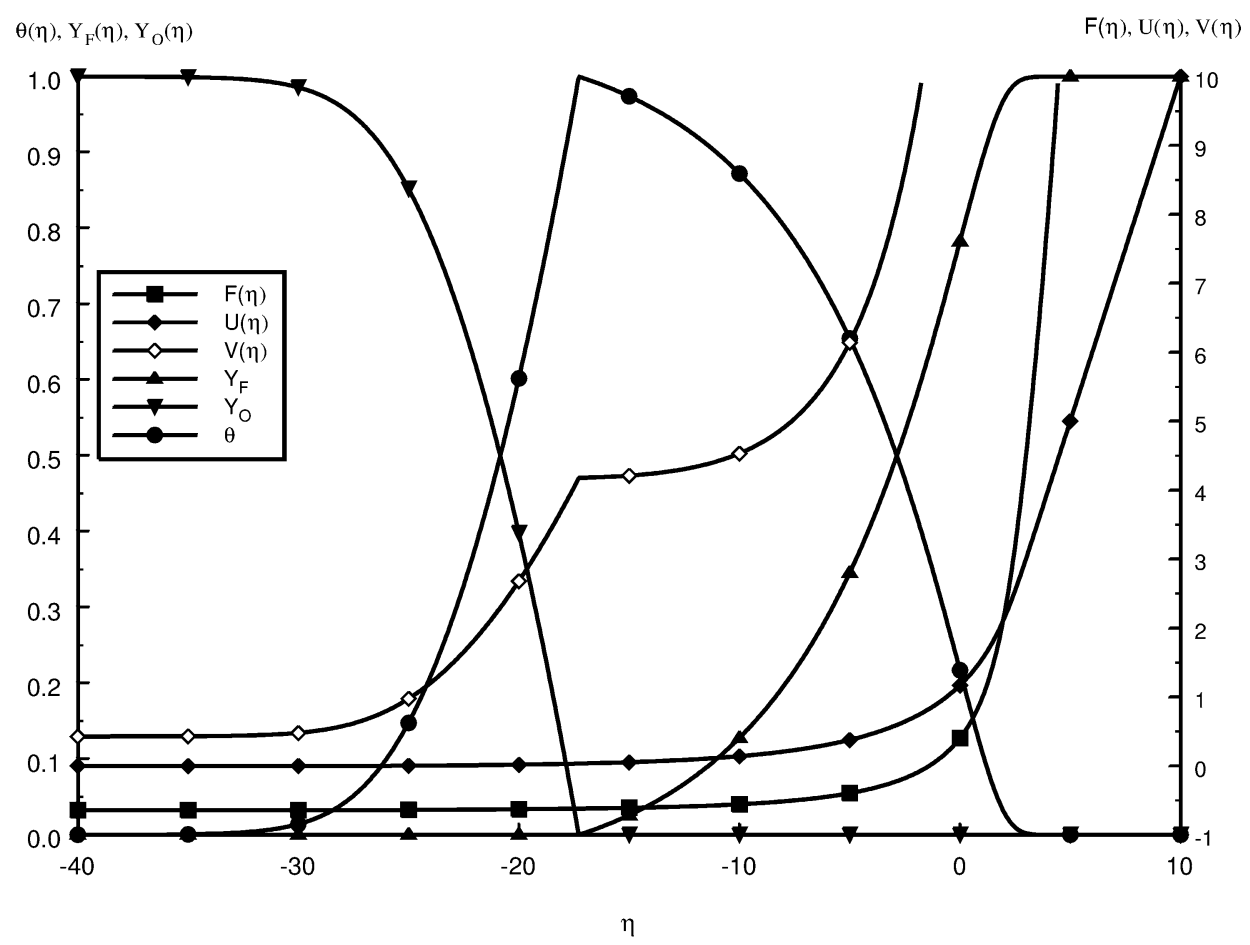

Fig. 2. Goldstein boundary layer self-similar solution, pure methane jet, $\gamma=8.5, S=17.4$.

values of $\xi, \tilde{F}(\xi \rightarrow \infty)=\xi+a$, where $a$ is to be determined in the course of the solution. Numerical solution of this problem gives $a=-1.58$, and $\tilde{F}^{\mathrm{II}}(0)=0.739$.

It is interesting that no very large changes are found in the value of the constant $C$ which appears in the generalized Goldstein boundary layer problem, when $S$ and $\gamma$ increase to fairly large values. For example, $C=-0.74$ and $\eta_{f}=-17.27$ were obtained for the pure methane jet $\left(Y_{F 0}=1, S=17.4\right)$ when thermal expansion is taken into account $(\gamma=8.5)$; while in the same case, when the effects of thermal expansion are not considered $(\gamma=0), C=-1.92$ and $\eta_{f}=-6.33$. If, in addition to neglecting thermal expansion, transport properties are taken as constant, we obtain $C=-1.17, \eta_{f}=-3.52$. For very diluted methane $\left(Y_{F 0}=0.1, S=1.74, \gamma=5.71\right)$ the results are $C=-0.80$, $\eta_{f}=-5.34$. Therefore, the far-field inviscid flow in the stagnant air appears to be determined mainly by the cold fuel jet.

The asymptotic form of the solution at large distances from the injector rim shows such slow decay of the perturbations that, as indicated before, they must be taken into account in the outer boundary conditions when solving numerically the conservation equations, in order to ensure accuracy in the results. Nevertheless, a large domain of calculation was required to ensure that the results are not dependent on the location of the boundary.

The numerical method used to solve the equations was based on standard second order central finite differences upon which Gauss-Seidel iterations were performed until the residuals decrease down to a sufficiently low predetermined level. A sensitivity analysis of the appropriate resid- uals level was performed in order to determine an optimal condition for the end of the iterations in terms of both computational cost and accuracy of the results; it was found that $\max \mid$ res $_{i, j} \mid<10^{-5}$ provides accurate solutions in acceptable computational time.

The selection of the calculation domain proved to be an important issue since non-appropriate boundaries would significantly change flame position. For the most demanding value of $S=17.4$, corresponding to a pure methane diffusion flame, the boundaries of the domain were varied from $y_{\text {min }}=-100$ to $y_{\text {min }}=-350$ and from $x_{\text {max }}=100$ to $x_{\max }=500$; the solution was found to be independent of the boundaries for $y_{\min } \leqslant-200$ and $x_{\max } \geqslant 200$, values that were used in most of the computations. Grid resolution effects were also tested for a pure methane jet diffusion flame for a moderately large Damköhler number $K a^{-1}=0.3$. In the diffusion flame front region, uniform grid spacing was used, while smooth uniform grid stretching was allowed out of this region. When using the values $\Delta x=\Delta y=1$, $0.5,0.25$ and 0.125 in the flame front region, no significant changes were found in the flame position or structure, although the overall heat transfer to the injector $q$ showed a more significant variation because, due to the stretching of the grid, resolution was not enough near the wall for the coarser grids. However, the error in heat transfer was lower than $3 \%$ when a uniform grid with $\Delta x=\Delta y=1$ was used, when compared with the finest grid. It should be noticed that due to the strong sensitivity of the solution to the value of the Karlovitz number near its critical flame lift-off value, this is not easy to determine. 


\section{Results}

Even if we limit the analysis of the structure of the attachment region of the diffusion flame to the case of a fuel jet in stagnant air we are left with a fairly large number of parameters. One of the parameters, $h_{p}=d_{p} / l_{N}$, is geometrical; in this paper we shall only consider the extreme cases $h_{p} \rightarrow 0$ and $h_{p} \rightarrow \infty$. In addition, we encounter the ratios of the diffusivities represented by the Lewis numbers, which in this paper we consider to be unity, and the Prandtl number that we assume to be $P r=0.72$.

Two important parameters are the thermochemical parameters $S=Y_{F 0} / s Y_{O 0}$ and $\gamma=Q Y_{F 0} / c_{p} T_{0}(1+S)$.

Finally we have a set of additional kinetic parameters, which we identify when $D$ is replaced in the reaction rate of Eq. (3) by its determination by (6) in terms of the effective Damköhler number $\mathrm{Ka}^{-1}$ (the main kinetic parameter), together with the non-dimensional activation energy $\beta$ and the reaction orders $n_{F}$ and $n_{O}$.

For a given fuel $n_{F}$ and $n_{O}$ are fixed, but the thermochemical parameters $S$ and $\gamma$ will change with the dilution of the fuel, or if we enrich the air with oxygen; in these cases $U_{L}$, and therefore the Damköhler number and $\beta$ will also change, for a fixed fuel velocity gradient.

Using the reaction rates proposed by Westbrook and Dryer [15], we have carried out calculations first for a pure methane jet in air for various Damköhler numbers $K a^{-1}$ for injectors with very thick walls or very thin walls. In addition, we have also analyzed the flame attachment region in the case where a jet of methane diluted with nitrogen emerges

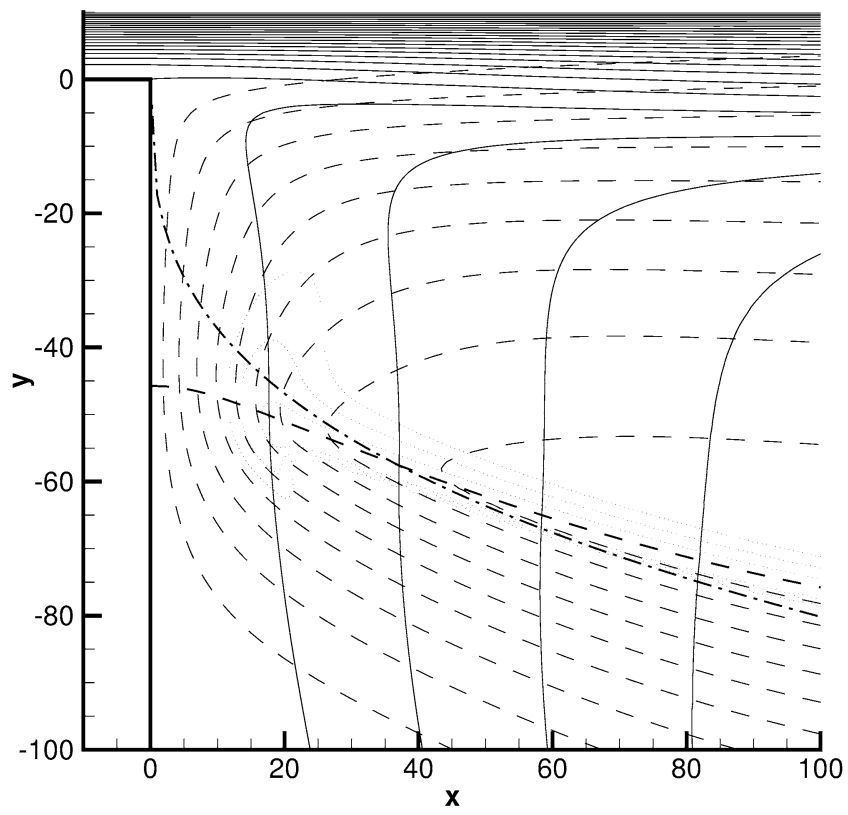

Fig. 3. Flow field for $Y_{F 0}=1.0$ and $\mathrm{Ka}^{-1}=1.0$, corresponding to point $\mathrm{A}$ in Fig. 9. Solid lines: stream lines, $\psi$ in intervals of 2.5, thin dashed lines: non-dimensional temperature $\theta=0.1$ to $\theta=0.9$, at intervals of 0.1 , thick dashed line: stoichiometric line, $Z=Z_{s}$, thick dot-dashed line: Goldstein flame position, dotted lines: non-dimensional reaction rate contours. from a wall in stagnant air (for various values of the methane mass fraction in the fuel stream).

Examples of the flow structure in the attachment region of the diffusion flames are shown in Figs. 3, 4 and 5 for a

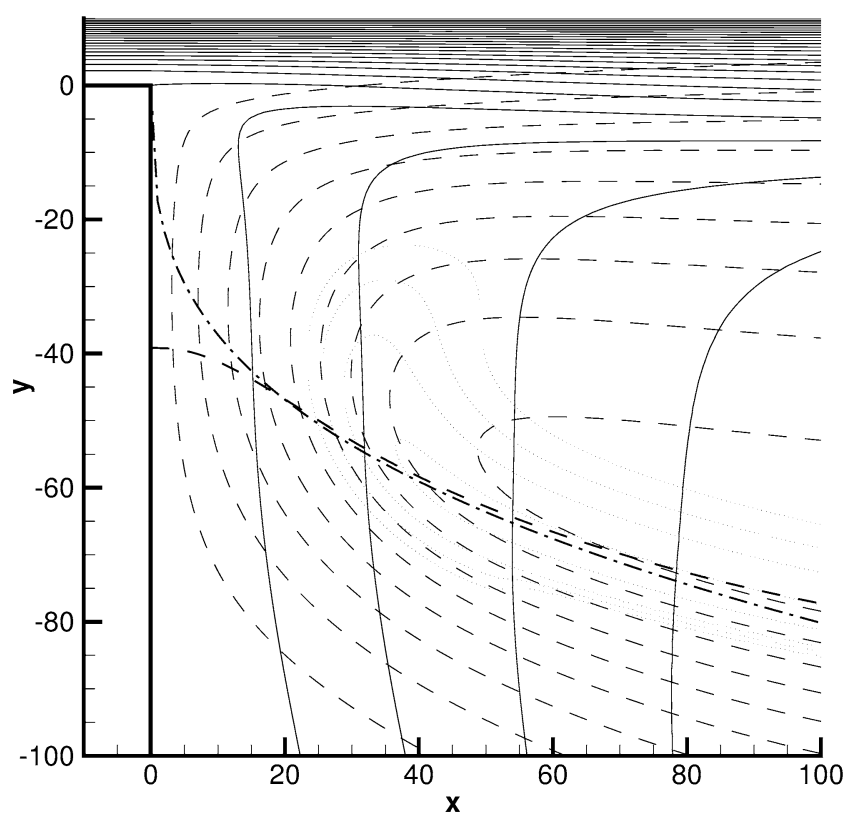

Fig. 4. Flow field for $Y_{F 0}=1.0$ and $K a^{-1}=0.04$, corresponding to point $\mathrm{B}$ in Fig. 9. Solid lines: stream lines, $\psi$ in intervals of 2.5, thin dashed lines: non-dimensional temperature $\theta=0.1$ to $\theta=0.9$, at intervals of 0.1 , thick dashed line: stoichiometric line, $Z=Z_{S}$, thick dot-dashed line: Goldstein flame position, dotted lines: non-dimensional reaction rate contours.

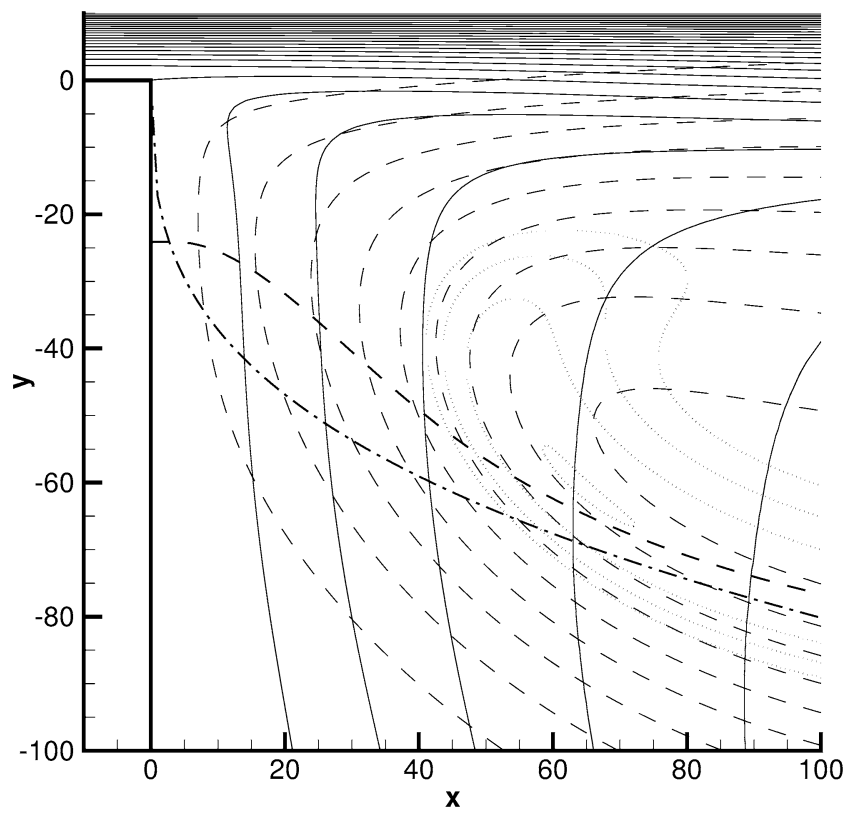

Fig. 5. Flow field for $Y_{F 0}=1.0$ and $\mathrm{Ka}^{-1}=0.019$, corresponding to point $\mathrm{C}$ in Fig. 9. Solid lines: stream lines, $\psi$ in intervals of 2.5, thin dashed lines: non-dimensional temperature $\theta=0.1$ to $\theta=0.9$, at intervals of 0.1 , thick dashed line: stoichiometric line, $Z=Z_{s}$, thick dot-dashed line: Goldstein flame position, dotted lines: non-dimensional reaction rate contours. 
pure methane jet issuing from a wall into stagnant air, for three values of the Karlovitz number, $K a=A \alpha_{0} / U_{L}^{2}$.

In Fig. 3 the effective Damköhler number $U_{L}^{2} / A \alpha_{0}$ is 1 and, hence, well above the lower critical Damköhler number for lift-off of the flame; in Fig. 5 the Damköhler number is 0.019 which is close to the critical value for lift-off. The flame structure in Fig. 3 shows a triple flame character of the type analyzed by Dold et al. [1] and Ruetsch et al. [11]. For lower values of the Damköhler number the flame edge is bent toward the fuel side and adopts the shape of a crook. For the lower value of $\mathrm{Ka}^{-1}$ shown in Fig. 5 the edge of the reaction zone has moved significantly away from the injector rim, so that the heat transferred to the wall has decreased strongly.

Notice that the resulting sizes of the flame attachment region reflected in the large values of the non-dimensional coordinates are large compared with the Navier-Stokes length $\left(\alpha_{0} / A\right)^{1 / 2}$. The reason is associated with the large value of the stoichiometric ratio $S=17.4$ for methane and the large value of the flame temperature rise, $\gamma=\left(T_{e}-T_{0}\right) / T_{e}=8.5$ for methane. The flame moves far into the originally stagnant air side of the flame to balance the weak entrainment convective flow with a higher thermal diffusivity.

In the figures we have shown the line where the mixture fraction has its stoichiometric value $Z=Z_{s}=1 /(1+S)$, and also the flame position when calculated with the generalization of the Goldstein solution.

When the calculations of the flame structure are repeated for cases in which the fuel jet is methane diluted with nitrogen, the flow structure changes significantly mainly

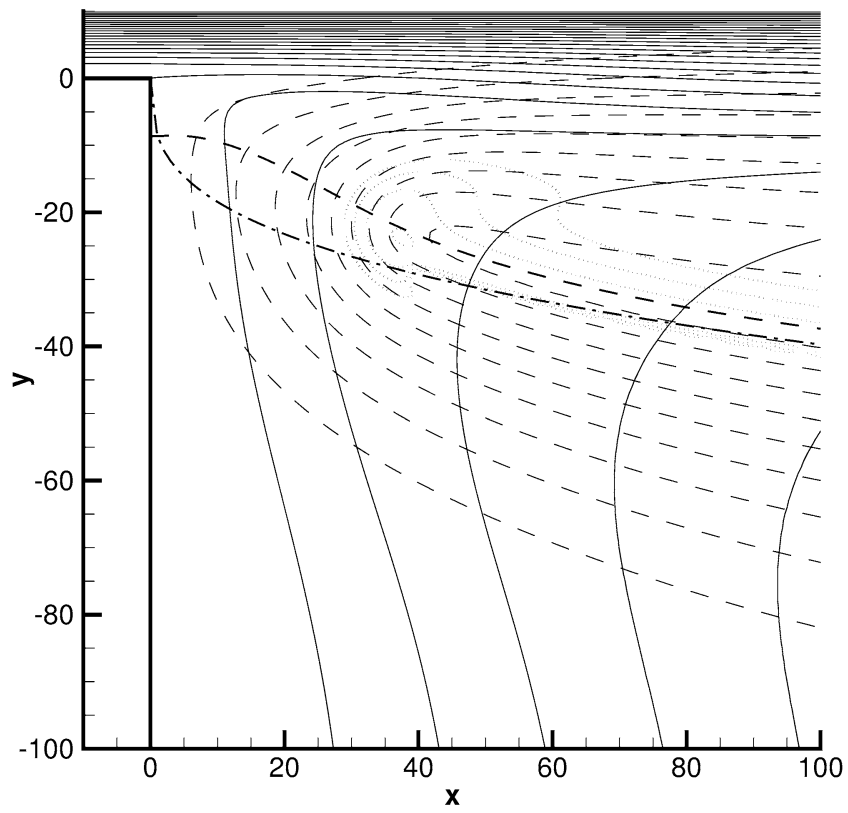

Fig. 6. Flow field for $Y_{F 0}=0.2$ and $\mathrm{Ka}^{-1}=0.08$, corresponding to point $D$ in Fig. 9. Solid lines: stream lines, $\psi$ in intervals of 2.5, thin dashed lines: non-dimensional temperature $\theta=0.1$ to $\theta=0.9$, at intervals of 0.1 , thick dashed line: stoichiometric line, $Z=Z_{s}$, thick dot-dashed line: Goldstein flame position, dotted lines: non-dimensional reaction rate contours. through the reduction of the air/fuel stoichiometric ratio, and also due to the (weaker) reduction in flame temperature. Some of the results are shown in Figs. 6 and 7, both corresponding to effective Damköhler numbers close to extinction. Notice the significant displacement of the flame

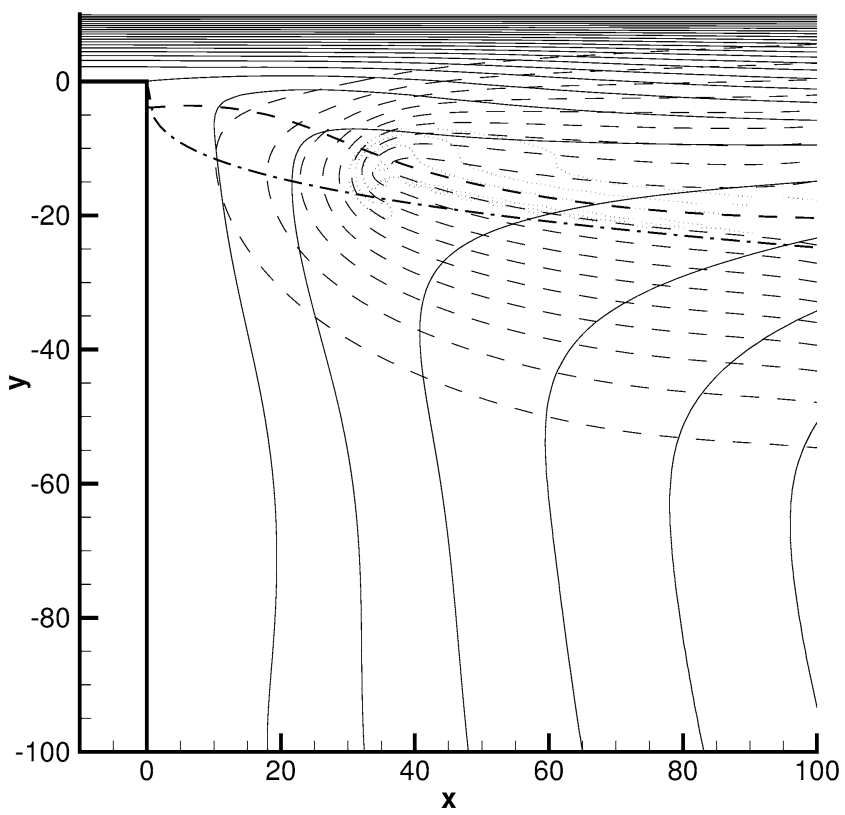

Fig. 7. Flow field for $Y_{F 0}=0.1$ and $K a^{-1}=0.18$, corresponding to point $\mathrm{E}$ in Fig. 9. Solid lines: stream lines, $\psi$ in intervals of 2.5, thin dashed lines: non-dimensional temperature $\theta=0.1$ to $\theta=0.9$, at intervals of 0.1 , thick dashed line: stoichiometric line, $Z=Z_{S}$, thick dot-dashed line: Goldstein flame position, dotted lines: non-dimensional reaction rate contours.

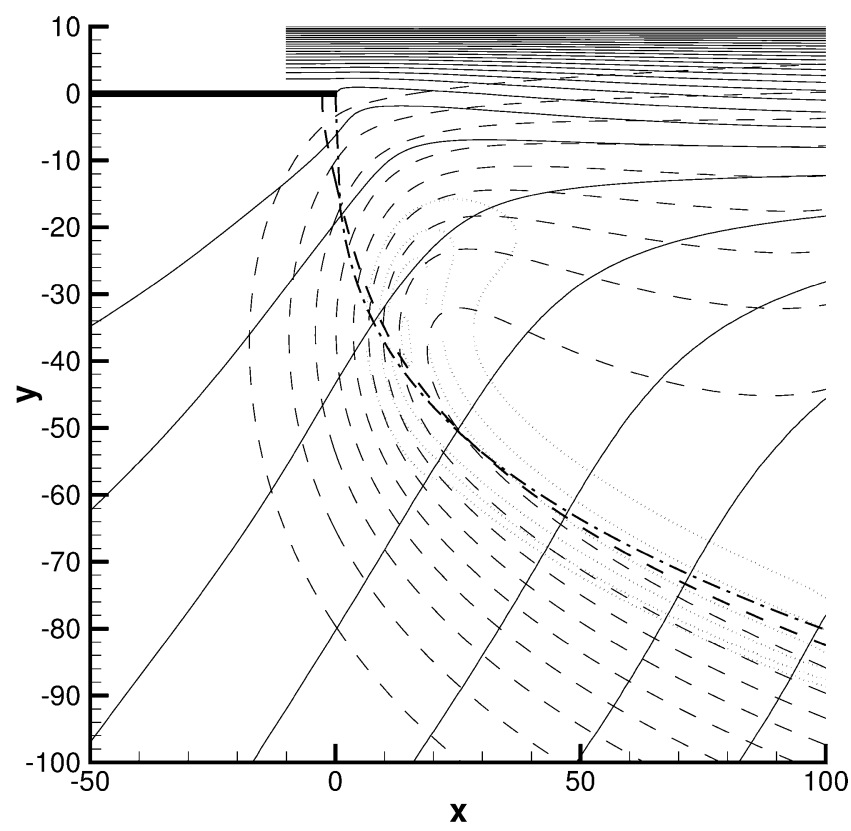

Fig. 8. Flow field for $Y_{F 0}=1.0$ and $\mathrm{Ka}^{-1}=0.04$, corresponding to point $\mathrm{F}$ in Fig. 9. Solid lines: stream lines, $\psi$ in intervals of 2.5, thin dashed lines: non-dimensional temperature $\theta=0.1$ to $\theta=0.9$, at intervals of 0.1 , thick dashed line: stoichiometric line, $Z=Z_{S}$, thick dot-dashed line: Goldstein flame position, dotted lines: non-dimensional reaction rate contours. 


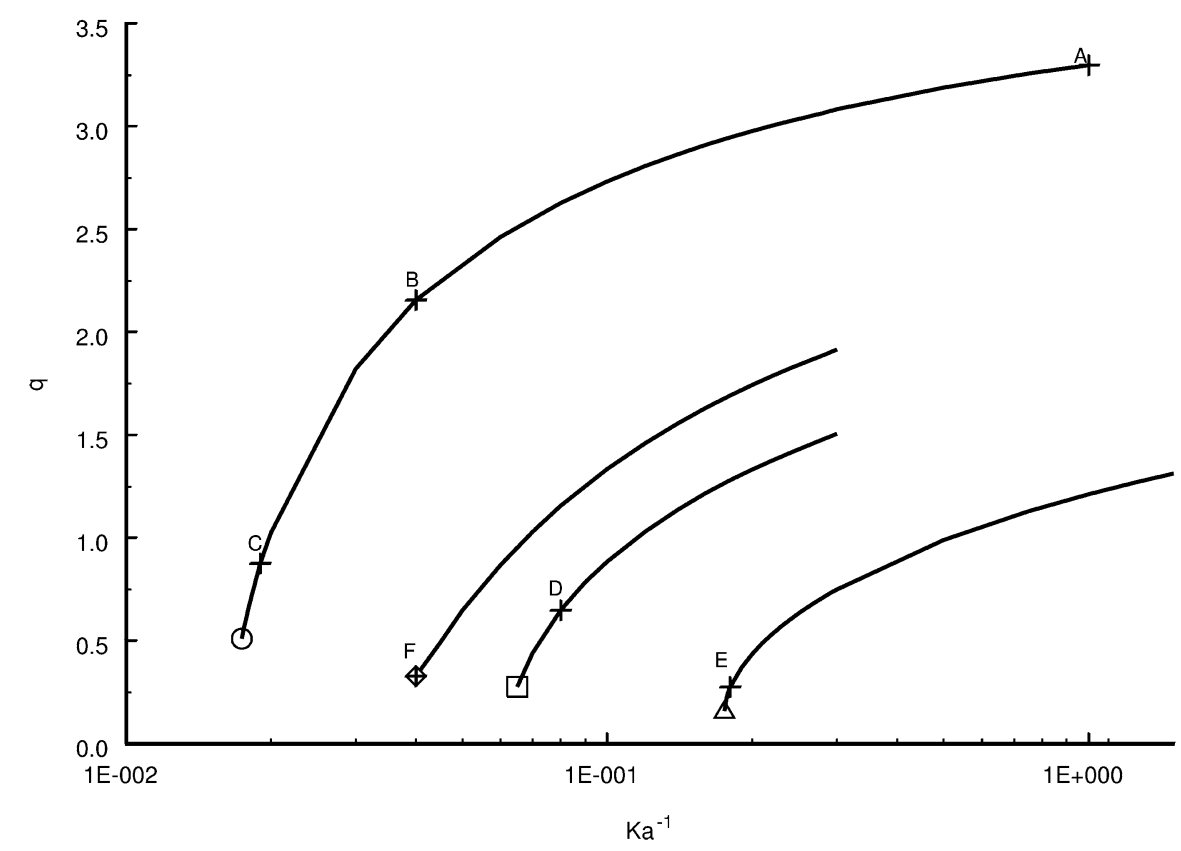

Fig. 9. Heat transfer to the wall as a function of inverse Karlovitz number. Circles: $Y_{F 0}=1, S=17.4, \gamma=8.5, \beta=8.0, h=\infty$, squares: $Y_{F 0}=0.2, S=3.48$, $\gamma=6.98, \beta=9.31, h=\infty$, triangles: $Y_{F 0}=0.1, S=1.74, \gamma=5.71, \beta=10.77, h=\infty$, diamonds: $Y_{F 0}=1, S=17.4, \gamma=8.5, \beta=8.0, h=0$.

toward the fuel stream when compared with the previous cases.

The difference between an infinite step and a separator plate can be seen in Figs. 4 and 8 where the flame structures are shown for $\mathrm{Ka}^{-1}=0.04$.

A summary of the results is given in Fig. 9 where the heat transferred by heat conduction to the injector $(q=$ $\left.\int_{\text {wall }} \frac{\partial \theta}{\partial n} d s\right)$, measured with the product $k_{0}\left(T_{e}-T_{0}\right)$ of fuel conductivity and rise in flame temperature, is shown in terms of the effective Damköhler number for various values of the jet dilution. The case of an infinitelly thin injector rim is also included. The heat flux to the injector decreases rapidly with decreasing values of $\mathrm{Ka}^{-1}$. No solution with an attached flame can be found for values of $\mathrm{Ka}^{-1}$ below a critical value, which increases with fuel dilution and is larger for a thin injector wall.

\section{Conclusions}

The region of attachment of diffusion flames to the rim of the fuel injector has been analyzed in the case where the fuel jet emerges into stagnant air.

The scale of the region of the flame attachment is determined by the wall value $A$ of the fuel velocity gradient, independently of the shape (parabolic or boundary layer type) of the fuel velocity profile if the Reynolds number is large enough. $A^{-1}$ is the residence time of the gas mixture in the region of flame attachment.

The flame gets attached to the injector if the Karlovitz number $A \alpha_{0} / S_{L}^{2}$ or the ratio of the chemical reaction time to the residence time in the planar premixed flame of the stoichiometric mixture (formed with 1 gram of the fuel stream and $S$ grams of air) is smaller than a critical value of the order of unity. The critical non-dimensional velocity gradient is larger for injectors with thick walls than for injectors with thin walls, so that the velocity gradient required to lift-off the flame is larger for thick walls than for thin walls. The critical Karlovitz number decreases with fuel dilution due to the lowering of the air/fuel mass stoichiometric ratio $S$.

The results are in qualitative agreement with the experimental results; due to the lack of information on the experimental value of the velocity gradient $A$ we can not make quantitative comparisons.

\section{Acknowledgements}

This work was supported by the Instituto Nacional de Técnica Aeroespacial (INTA) of the Spanish Ministry of Defense, under the Investigation Programme IGB 4400903. V.K. acknowledges the support of CONACYT (Mexico) through a 'Cátedra Patrimonial nivel III' fellowship. The work of A.L. was supported by the Ministry of Science and Technology under project BFM 2001-3691.

\section{References}

[1] J.W. Dold, L.J. Hartley, D. Green, Dynamical Issues in Combustion Theory, Fife, in: P.C. Fife, A. Liñán, F.F. Williams (Eds.), in: IMA Volumes in Mathematics and Its Applications, Vol. 35, Springer, 1991, pp. 107-126.

[2] E. Fernández-Tarrazo, V.N. Kurdyumov, A. Liñán, Diffusion flame attachment and lift-off in the near wake of a fuel injector, Proceedings of the Combustion Institute 28 (2000), to appear. 
[3] A.G. Gaydon, H.G. Wolfhard, Flames, their Structure, Radiation and Temperature, Chapman \& Hall, 1953.

[4] S. Goldstein, Concerning some solutions of the boundary layer equations in hydrodynamics, Proc. Cambridge Philos. Soc. 26 (1930) $1-30$.

[5] F.J. Higuera, A. Liñán, Flow field of a diffusion flame attached to a thick-walled injector between two coflowing reactant streams, J. Fluid Mech. 329 (1996) 389-411.

[6] T. Kawamura, K. Asato, in: Proc. 2nd. European Symposium on Combustion, 1975, pp. 611-616.

[7] T. Kawamura, K. Asato, T. Mazaki, Combustion Science and Technology $22(1980) 211-216$

[8] A. Liñán, Ignition and flame spread in laminar mixing layers, in: Combustion in High Speed Flows, Kluwer Academic, Dordrecht, 1994, pp. 461-476.
[9] A.F. Messiter, Boundary-layer flow near the trailing edge of a flat plate, SIAM J. Appl. Math. 18 (1) (1970) 241-257.

[10] K. Robson, M.J.G. Wilson, Combustion and Flame 12 (1969) 626 634.

[11] G.R. Ruetsch, L. Vervisch, A. Liñán, Effects of heat release on triple flame propagation, Physics of Fluids 7 (6) (1995) 2496-2503

[12] K. Stewartson, On the flow near the trailing edge of a flat plate II, Mathematika 16 (1969) 106-121.

[13] F. Takahashi, W.J. Schmoll, Lifting criteria for jet diffusion flames, Proceedings of the Combustion Institute 23 (1990) 677-683.

[14] F. Takahashi, W.J. Schmoll, V.R. Katta, Proceedings of the Combustion Institute 27 (1998) 675-684.

[15] C.K. Westbrook, F.L. Dryer, Simplified reaction mechanisms for the oxidation of hydrocarbon fuels in flames, Combustion Science and Technology 27 (1981) 31-43. 